\title{
La psicología conductista a la luz de los programas de investigación científica de Imre Lakatos
}

\author{
Ramiro Gutiérrez Vásquez ${ }^{1, a}$
}

\section{RESUMEN}

El análisis epistemológico de la Psicología Conductista es hecho tomando como modelo de análisis la propuesta de Imre Lakatos conocida como la "Metodología de los Programas de Investigación Científica" (MPIC). Se describe la estructura de las teorías científicas en la versión de Lakatos para luego contrastarla con el modelo conductual de SKINNER, quien sostiene que las "contingencias de reforzamiento" van moldeando y determinando el comportamiento de los organismos, incluido los humanos. Se analiza el método y la forma en que han desarrollado el conocimiento de los principios y las generalizaciones de comportamiento a partir de estudios con animales de laboratorio, que dieron origen al Análisis Experimental del Comportamiento. El problema de la validez de los enunciados o proposiciones del lenguaje conductista, y el problema de la naturaleza de sus predicciones son vistos a la luz de la concepción de LAKATOS. Luego del análisis, se establece que los cambios conceptuales o el uso de las nuevas categorías para describir los principios generales de comportamiento, deben ir acompañadas de una revisión de sus fundamentos filosóficos. El análisis termina ensayando una respuesta a la siguiente pregunta: ¿El Análisis Experimental de la Conducta (AEC) cumple con los requisitos para ser considerado como un "Programa de Investigación Científica”, según Lakatos?

PALABRAS CLAVE: Metodología de los programas de investigación científica, análisis epistemológico, principios conductuales, análisis experimental.

\section{SUMMARY}

The epistemological analysis of Behavioural Psychology is made on the model of the proposed analysis of Imre Lakatos known as the "Methodology of Scientific Research Programs" (MPIC). It describes the structure of scientific theories in the version of Lakatos and then contrast it with the behavioral model of Skinner, who argues that the "contingencies of reinforcement" are shaping and determining the behavior of organisms, including humans. It is analized the the method and the way they have developed knowledge of behavioral principles and generalizations from studies with laboratory animals, that originated the Experimental Analysis of Behavior. The problem of validity of the statements or propositions of behavioral language and the problem of the nature of their predictions are seen in the light of the conception of Lakatos. After the analysis, it is established that the conceptual changes or the use of new categories of behavior to describe the general principles of behavior, must be accompanied by a review of its philosophical foundations. The analysis ends rehearsing an answer to the following question: ¿Does the Experimental Analysis of Behavior (AEC) meet the requirements to be listed as a "Scientific Research Program" according to Lakatos?

KEYWORDS: Methodology of scientific research programs, epistemological analysis, behavioral principles, 
experimental analysis.

\section{INTRODUCCION}

La ciencia contemporánea ha tenido un gran impulso y auge a raíz de la novísima revolución en las ciencias naturales, que surgió a mediados de la década del 90 del siglo XIX y que continúa a la fecha. Entre el inmenso número de adelantos científicos, se pueden destacar, en especial los que permitían a la ciencia el acceso a las dos partes principales del átomo: el núcleo y la capacidad electrónica. Los descubrimientos de la radiactividad y del radio llevaban al conocimiento del núcleo y las transformaciones nucleares; los del electrón y los rayos roentgen al conocimiento de la capa.

Hoy en día, según Kedrov, la novísima ciencia natural está en su tercera etapa de desarrollo, es decir, está ligada con los descubrimientos de la física nuclear, comenzó en vísperas de la segunda guerra mundial con el descubrimiento del neutrón y en especial, con el de la fisión de los núcleos pesados (uranio) quién sentó el comienzo de la época de la energía atómica. Aún esta etapa no ha concluido, no obstante, dice KEDROV, que se vislumbran los contornos de la cuarta etapa, relacionada con la penetración de la física en las profundidades de las propias partículas elementales: protón, neutrón, mesones (Kedrov, 1977).

En biología ha sucedido otro tanto. Desde la teoría de la evolución de Darwin, esta ciencia de la vida, ha emprendido un despliegue revolucionario sólido. $\mathrm{Su}$ primera consecuencia la sufrió la teoría creacionista del hombre. La versión metafísica de la vida y sus principios legales teológicos y vitalistas, fueron desplazados por principios explicativos más objetivos y racionales. Otra consecuencia constructiva de esta teoría ha sido la elaboración de nuevas ciencias, como la biología molecular cuya fuerza explicativa sobre los mecanismos de la vida se encuentran plasmados en el modelo del ADN elaborado por Watson y Crick. Este modelo molecular de la vida es ingeniosamente aplicado al estudio de la solución de problemas del ser humano a través de la ingeniería genética. Actualmente esta ciencia ha evolucionado hasta tal punto que se cuenta con información sobre la descripción de toda la secuencia de replicación del ADN, gracias al proyecto sobre el genoma humano dirigido por Pray (2008).

Las ciencias sociales también han visto operar en su ámbito cambios sustanciales, es decir, una nueva manera de abordary transformar los fenómenos sociales que han originado disciplinas contemporáneas. Estas disciplinas ya han adquirido un carácter de ciencia genuina y ambicionan tener éxito en la descripción, explicación y un acercamiento predictivo de las formas y mecanismos de interrelación, a diferentes niveles, entre los hombres.

No sólo se han producido revoluciones científicas en la física, en las ciencias biológicas o ciencias sociales, arriba mencionadas brevemente, también en las ciencias psicológicas los cambios teóricos y conceptuales para comprender, y hasta explicar el psiquismo humano se han visto innovados. Para algunos autores, la psicología adquiere su carácter de ciencia a partir de Wundt y el establecimiento del primer laboratorio experimental (Piaget, 1978). Para otros autores, es Iván Pavlov con el descubrimiento de los reflejos condicionados, quién instaura el carácter de ciencia de la psicología. Lo cierto es que se necesita hacer una serie de investigaciones a través de las metodologías que nos brindan los modelos epistemológicos y la historia de la ciencia crítica a fin de responder a una importante pregunta, que desde mi punto de vista, aún no ha sido contestada con objetividad y rigor, a saber: ¿desde cuándo y con qué acontecimiento o hecho psicológico la psicología adquiere su rango de ciencia? (Robinson, 1985).

La Psicología es estudiada por diferentes sistemas: el Estructuralismo de Wundt, el Funcionalismo de William James, el Psicoanálisis, pasando por el Conductismo, el Cognitivismo, la escuela Piagetiana, la propuesta Socio-Cultural de Vygotsky, el Humanismo de Rogers y Maslow hasta la perspectiva Dialéctica de la Psicología (Robinson, 1985).

De todas estas variantes explicativas de los fenómenos psíquicos parece ser que los trabajos del conductismo y los trabajos de los psicólogos orientados con fundamentos dialécticos e históricos están más comprometidos con el quehacer del mundo científico.

Precisamente éste trabajo desarrolla el análisis epistemológico de la alternativa conductista basado en el paradigma de los "Programas de Investigación Científica" elaborado por Imre Lakatos (1978). Si bien es cierto que existen algunos trabajos que abordan la problemática epistemológica al interior del conductismo, el común denominador de estos trabajos es que no abordan el estudio de la psicología conductista pertrechados de modelos epistemológicos como los de Popper (1978;1980), Kuhn (1977), Piaget (1978) o de la Dialéctica de Mikulinski (1977), entre otros. Parece que el sustento epistémico de la psicología 
conductista está basado en el positivismo lógico, en consecuencia, se podría argüir en contra de la validez de nuestro trabajo, el carácter de inconmensurabilidad de los paradigmas. Sin embargo, no será obstáculo para penetrar al interior del trabajo de la psicología conductista, es más, pensamos que si sus postulados conceptuales son sólidos saldrá victoriosa del examen al cual será sometido.

Este trabajo de investigación se ha trazado varios objetivos:

Discutir el estatus de ciencia de la psicología conductista teniendo como parámetro de contraste a los "Programas de Investigación Científica" de Lakatos.

Demostrar que la crisis en que se encuentra el sistema psicológico conductista, se debe fundamentalmente a sus presupuestos filosóficos, antes que a su sistema conceptual elaborado.

Demostrar que el marco de investigación de la psicología conductista tiene serias limitaciones en cuanto a la predictibilidad de los hechos psicológicos.

Es posible lograr ciertos niveles de conmensurabilidad entre paradigmas epistemológicos y psicológicos.

Se ha creído conveniente dividir el contenido del trabajo en varias partes. Bajo el sub título de "Epistemología y Psicología Conductista" queremos presentar la estructura y los mecanismos que Lakatos ha denominado "Programas de Investigación Científica", acompañado de los criterios que para él permitirían deslindar entre lo científico de lo pseudo científico. Luego pasaremos al análisis experimental de la conducta desde los puntos de vista de su filosofía, de su labor científica y de su tecnología a fin de tener un panorama claro e integral de su quehacer psicológico. En el siguiente paso se hará el estudio al interior de la psicología conductista teniendo como paradigma de contraste a los Programas de Investigación Científica (PIC) de Lakatos.

\section{Epistemología y psicología conductista}

Bajo este título se quiere dar a conocer tres problemas fundamentales: una aproximación sobre significado del paradigma epistemológico lakatosiano, luego un abordaje al interior del trabajo de la psicología conductista y finalmente el examen crítico-epistémico sobre la naturaleza de cientificidad de este modelo psicológico.

\section{Los programas de investigacion cientifica (PIC)}

\section{Ciencia y pseudociencia en los PIC}

Varias son las alternativas que se han propuesto a lo largo de la Historia de la Ciencia y de la Filosofía a la pregunta ¿Qué distingue al conocimiento científico de la ignorancia y a la ciencia de la pseudociencia?. Una de las alternativas fue propuesta por los seguidores de la lógica inductiva, para quienes la fuerza de una teoría científica estaría en función de su probabilidad matemática: si esta probabilidad es elevada se califica a la teoría como científica; si la probabilidad es baja o cero, la teoría es calificada como no científica. Así, esta propuesta nos suministra una especie de escala o gradaciones continuas que van desde las teorías débiles, de baja probabilidad, hasta las teorías poderosas, de alta probabilidad.

Por supuesto este criterio del probabilismo no tuvo mucha fortuna. Ya en 1934 Popper enfiló duras críticas a este pensamiento lógico inductivo. Karl Popper (1980) uno de los filósofos de la ciencia más destacados e influyentes de nuestro siglo, sostuvo que la probabilidad matemática de todas las teorías científicas o pseudo científicas, para cualquier magnitud de evidencia es cero. En otras palabras, las teorías científicas no sólo son incapaces de ser probadas, sino que también son igualmente improbables. Popper, prácticamente invalidó con su argumentación el carácter científico de las teorías construidas a partir de la inducción.

Propuso un criterio de demarcación diferente con el fin de evitar el "error positivista" cuyo criterio propuesto eliminaba los sistemas teóricos de la ciencia natural; este error para Popper, se evita eligiendo un criterio que permita admitir en el dominio de la ciencia empírica, incluso, enunciados que no pueden verificarse; en otras palabras, una teoría puede ser científica incluso si no cuenta ni con una pizca de evidencia favorable, y puede ser seudocientífica aunque tenga toda la evidencia a su favor. Es más, el carácter científico o no de una teoría puede ser determinado con independencia de los hechos. Al respecto el propio Popper nos habla sobre el asunto “... debemos de adoptar no el criterio de la verificabilidad, sino el de la falsabilidad de los sistemas, no exigiré que un sistema científico pueda ser seleccionado, de una vez para siempre, en un sentido positivo; pero sí que sea susceptible de selección en un sentido negativo por medio de contrastes o pruebas empíricas: ha de ser posible refutar por la experiencia un sistema científico 
empírico" (Popper,1980).

Por consiguiente, Popper trata de resolver, con su criterio de refutabilidad, no un problema de sentido o significación, ni tampoco un problema de verdad o aceptabilidad, "sino el de trazar una línea demarcatoria (en la medida en que esto pueda hacerse) entre los enunciados o sistemas de enunciados que sean de carácter religioso o metafísico, o simplemente pseudo científico para ser colocados en el rango de científicos $y$, los enunciados o sistemas de enunciados que deben ser suceptibles de entrar en conflicto con observaciones posibles o concebibles" (Popper,1980).

Entonces, ¿quiere decir que el criterio de falsabilidad de Popper es la solución al problema de demarcación entre la ciencia y la pseudociencia? Ante esta pregunta Imre Lakatos (contesta negativamente, ya que para él, por ejemplo, el criterio de Popper no toma en cuenta "la tenacidad de las teorías científicas", menos aún, que los científicos abandonen una teoría simplemente porque los hechos la contradigan. En tal sentido, para Lakatos, la historia de la ciencia está, por supuesto repleta de exposiciones sobre cómo los experimentos cruciales supuestamente destruyen a las teorías. Pero tales exposiciones suelen estar elaboradas mucho después de que la teoría haya sido abandonada. $\mathrm{Si}$ Popper, continua Lakatos, hubiera preguntado a un científico newtoniano en qué condiciones experimentales abandonaría la teoría de Newton, algunos científicos newtonianos hubieran recibido la misma calificación que algunos marxistas (Lakatos, 1974).

¿Qué es entonces lo que distingue a la ciencia? o tenemos que aceptar la propuesta de Thomas Kuhn, quien llegó a la conclusión que una revolución científica sólo es un cambio irracional de convicciones, al estilo de una conversión religiosa (Kuhn, 1977). Pero si se acepta el razonamiento de Kuhn, dice Lakatos, no existe una demarcación explícita entre ciencia y pseudociencia, ni distinción entre progreso científico y decadencia intelectual: no existe un criterio objetivo de honestidad (Lakatos, 1978).

Si los paradigmas epistemológicos, hasta ahora descritos brevemente, no satisfacen las exigencias requeridas para decidir sobre el carácter científico de un sistema de enunciados, entonces ¿qué criterios se puede optar para distinguir entre lo científico y lo no científico?

Para dar respuesta a esta interrogante nos basaremos en la propuesta de la metodología de los programas de investigación científica (MPIC) propuesta por
ImreLakatos, un ingenioso discípulo de Popper, quien en todas sus obras ha tratado de desarrollar los criterios del racionalismo crítico poppneriano o de elaborar, lo que él denomina, las reconstrucciones racionales de la ciencia (Lakatos, 1974).

Según Lakatos, los acontecimientos científicos no son hipótesis aisladas, sino que la unidad descriptiva típica es un Programa de Investigación. No se puede afirmar que la Ciencia sea tan sólo una serie de ensayos y errores, o, para hablar en lenguaje popperiano, no es una serie de conjeturas y refutaciones (Lakatos, 1974). El ejemplo de Popper de que la proposición todos los cisnes son blancos puede ser falseada por el descubrimientos de un cisne negro, son para Lakatos casos triviales de ensayo y error que no forman la ciencia (Popper, 1980).

La ciencia para Lakatos, se desarrolla en base a Programas de Investigación (Lakatos, 1978). A diferencia de Popper, propone que la distinción entre un programa científico progresivo y otro pseudocientífico o regresivo, no puede radicar en que algunos aún no han sido refutados, sino reside en la característica de predecir hechos nuevos, hechos que ni siquiera previamente habían sido soñados o que incluso habían sido contradichos por programas previos rivales. Lakatos nos remite, por ejemplo, a la predicción de Halley, quien trabajó en el programa científico de Newton sobre la nueva aparición del cometa (que ahora lleva su nombre) en el firmamento terrestre después de 72 años, hecho que fue corroborado. O bien, el programa de Einstein quien predijo de que sí se mide la distancia entre dos estrellas por la noche y sí se mide la misma distancia de día (durante un eclipse de sol) las dos mediciones serán distintas. Esta predicción también fue corroborada años después de haberse planteado como hipótesis. Por lo tanto, el distintivo del progreso empírico no son las verificaciones triviales (Lakatos, 1978).

No es un éxito para la teoría newtoniana el que al soltar una piedra ésta caiga hacia la tierra, sin que importe el número de veces que se repite el experimento. Pero las llamadas refutaciones no indican un fracaso empírico como Popper ha enseñado, porque todos los programas crecen en un océano permanente de anomalías. Lo que realmente importa son las predicciones dramáticas, inesperadas, grandiosas; unas pocas de estas son suficientes para decidir el desenlace; sí la teoría se retrasa con relación a los hechos, ello significa que estamos en presencia de programas de investigación pobres y regresivos 
(Lakatos, 1978).

En contra de la propuesta popperiana, la de Lakatos nos ofrece racionalidad inmediata, ya que puede ocurrir que un programa adquiera su carácter progresivo (científico) después de varios años y deje de ser regresivo (no científico). Las críticas importantes son constructivas, es decir, los programas de investigación progresivos sustituyen a los regresivos.

\section{Estructura y dinamica de los PIC}

La metodología de los programas de investigación científica posee también un carácter demarcacionista, es decir, constituye una definición de progreso científico universal. Hablemos sobre sus características.

Consiste en una serie de teorías en desarrollo que tiene una estructura: Posee un núcleo firme persistente, como es el caso de las tres leyes de la mecánica y la ley de la gravitación en el programa de Newton, o como el caso de la ley de asimilación, acomodación y equilibración del programa de Piaget.

También está conformado, por un gran "cinturón protector" de hipótesis auxiliares sobre cuya base se establecen las condiciones iniciales. Este cinturón protector protege al núcleo firme y es susceptible de ser sometido a refutaciones, en realidad son como hipótesis auxiliares.

Por ello, en parte, a la fuerza de los hechos, el cinturón protector es modificado, expandido, complicado, mientras que el núcleo firme permanece intacto. En el programa de Newton este papel protector correspondía a la óptica geométrica, a la refracción atmosférica, entre otros.

Además, los programas de investigación cuentan con una heurística conformada por un conjunto de técnicas para la solución de problemas. Por ejemplo, para el caso de Newton la heurística fue su aparato matemático: cálculo diferencial, la teoría de convergencia y las ecuaciones integrales y diferenciales.

Ahora bien, una vez planteada la estructura de los PIC ¿Cómo es que se evalúan estos programas?, ¿Cuál es su mecanismo de acción?

Lakatos sostiene que un programa de investigación es progresivo o regresivo. Tiene un progreso técnico en el caso de que cada modificación conduzca a nuevas e inesperadas predicciones, y es empíricamente progresivo si algunas de tales predicciones nuevas resultan corroboradas. El científico puede solucionar anomalías realizando ajustes adecuados en su programa, maniobras que son ad hoc (Lakatos, 1978). También existe en la metodología de los PIC una noción de progreso heurístico: consiste, dice Lakatos, en que las sucesivasmodificaciones del "cinturón protector" deben ser acordes con la heurística. Los científicos desconfían, acertadamente, de los procedimientos ad hoc para la solución de anomalías. Un PIC supera a otro si tiene un exceso de contenido de verdad sobre su rival, en el sentido de que predice más cosas que su rival, y algunos casos adicionales. La heurística es clasificada por Lakatos como negativa y positiva.

En los PIC el modus tollens se dirige contra el cinturón protector y no contra el núcleo firme gracias a la acción de la heurística negativa, es decir, las hipótesis auxiliares deben recibir los impactos de las contrastaciones, y para defender al núcleo firme, será ajustado y reajustado e incluso completamente sustituido. Para Lakatos, un programa de investigación tiene éxito si ello conduce a un cambio progresivo de problemática; fracasa, si conduce a un cambio regresivo (Lakatos, 1978).

Por ejemplo, en el programa de Newton la heurística negativa impide dirigir el modus tollens contra las tres leyes de la dinámica y contra la ley de la gravitación. Este núcleo firme es irrefutable por decisión metodológica de sus defensores; las anomalías solo permiten originar cambios en el cinturón protector de hipótesis auxiliares observacionales y en las condiciones iniciales. Cabe aclarar que según Lakatos, el auténtico núcleo firme del programa realmente no nace ya dotado de toda su fuerza, sino que se desarrolla lentamente mediante un proceso largo, preliminar de ensayos y errores.

Los PIC también operan con una heurística positiva además de la heurística negativa. Mientras que la heurística negativa especifica el núcleo firme del programa que es irrefutable, por decisión metodológica de sus defensores; la heurística positiva consiste en un conjunto, parcialmente estructurado, de sugerencias o planes sobre cómo cambiar y desarrollar las versiones refutables del programa de investigación, es decir, nos conduce al mecanismo de como modificar o complicar el cinturón protector que está siendo refutado.

La heurística negativa opera impidiendo que el científico se pierda en el océano de anomalías. La heurística positiva opera estableciendo una diversidad de modelos crecientemente complicados que se acerquen cada vez más a la realidad. Así, el trabajo 
del científico se centra en la construcción de sus modelos siguiendo las pautas establecidas en la parte positiva de su programa. En consecuencia, ignora los contraejemplos reales, los datos disponibles.

Estas consideraciones nos conducen a deducir que la heurística positiva se arraiga casi sin tener en cuenta las refutaciones; puede parecer también que son las verificaciones y no las refutaciones las que suministran los puntos de contacto con la realidad. Aunque, dice Lakatos, se debe señalar que cualquier verificación de la versión $(n+1)$ del programa es una refutación de la versión, no podemos negar que algunas derrotas de las versiones sub siguientes, siempre son previstas; son las verificaciones las que mantienen la marcha del programa (Lakatos, 1978).

Por lo tanto, la selección racional de problemas que realizan los científicos, según Lakatos, está determinada por la heurística positiva del programa y no por las anomalías psicológicamente embarazosas. Las anomalías se enumeran pero se archivan después en la esperanza de que, llegado el momento se convertirán en corroboraciones del programa. Es más, Lakatos sostiene que aquellos científicos que trabajan en ensayo y error o en una fase degenerada del programa de investigación cuya heurística positiva se quedó sin contenido, se ven obligados a redoblar su atención a las anomalías.

\section{Sobre la predicción en los PIC}

En los programas de investigación propuestos, la predicción está ligada al lado progresivo del programa, es decir, mientras su desarrollo teórico anticipa su desarrollo empírico, mientras continúa prediciendo nuevos hechos con cierto éxito (Lakatos, le llama problemática progresiva y dentro de ella trata sobre la predicción)(Lakatos, 1978). Por el contrario, el carácter no predictivo del programa científico se relaciona al lado estancado; es decir, cuando el desarrollo teórico queda rezagado respecto de su desarrollo empírico; esto es, cuando solo aduce explicaciones post hoc, o bien sólo proporciona descubrimientos por casualidad, o produce hechos anticipados por y descubiertos en un programa rival.

Según estas consideraciones, un PIC con más fuerza predictiva reemplaza a otro, es decir, la predicción está en relación directa con el carácter progresivo en la explicación del PIC rival; sí el PIC se encuentra relacionado más directamente con su carácter estancado no conducirá a reales predicciones.
En conclusión, la fuerza predictiva, o el PIC "progresivo" determinará una especie de línea demarcatoria entre la teoría científica y la teoría marcada por el PIC como "estancada", pero tan sólo desde un punto de vista temporal, ya que pudiera darse cambios en las condiciones de la formulación ingeniosa de nuevas hipótesis auxiliares o Ad hoc y entonces lo "estancado" se convertirá en "progresivo" o viceversa.

A todo esto Lakatos (1978) nos recuerda que "es muy difícil decidir, ya que no se debe exigir progreso en cada paso, cuándo un PIC se ha estancado definitivamente, o cuándo uno de los programas rivales ha conseguido una ventaja decisiva sobre éste. En esta metodología no puede darse una racionalidad instantánea (cuando menos mecánica), ni el veredicto de anomalía, por parte del científico experimental, pueden anular un PIC de un sólo golpe. Sólo se puede ser adivino después del evento".

\section{ACERCA DEL CONDUCTISMO}

\section{El conductismo radical}

En uno de los artículos teóricos: El conductismo a los cincuenta, Skinner (Pardo y natalicio, 1975) define al conductismo como la filosofía de la ciencia que se ocupa del contenido y los métodos de la psicología. Más tarde, en su libro sobre el conductismo, de carácter filosófico, distingue dos variantes al interior del conductismo, producto de estilos metodológicos diferentes para abordar el estudio de la conducta observable, objeto del estudio de la Psicología. Estas variantes se conocen con el nombre de: Conductismo metodológico y Conductismo Radical.

Sostuvo que la diferencia más resaltante entre ambas opciones metodológicas se encuentra en el modelo epistemológico que usan como estrategia para estudiar el comportamiento de los organismos: el Conductismo Metodológico utiliza un modelo E $\mathrm{O} R$, enfatizando en variables orgánicas, es decir, en los procesos y estados fisiológicos al interior del sujeto (se le conoce como variable "O"). La variable estímulo (E) es una suerte de input cibernético que origina ciertos cambios dentro del sujeto, cambios que se elaboran en $(\mathrm{O})$ para luego emitir una respuesta (R). El segundo modelo o Conductismo Radical no tiene en cuenta la variable "O" para el análisis del comportamiento, se realiza el análisis de la conducta a partir de eventos "observables", "medibles": los estímulos discriminativos (ED), las respuestas (R) 
y los estímulos reforzadores (ER) no necesitan ser constructos hipotéticos, sino que tienen un sustento empírico directo extraído de los hechos conductuales. Esta nueva manera de estudiar el comportamiento de los organismos se le denomina "Análisis Experimental de la Conducta". Es decir, a la conducta global se le analiza en sus componentes más simples y a cada componente se le investiga por separado a fin de conocer las "causas" que la determinan.

En conclusión, esta peculiar manera de abordar la investigación psicológica cuenta con un aval filosófico (el conductismo radical), y con ciertos principios, procedimientos y métodos (como prácticas científicas) que se aplican, sobre todo, a nivel de laboratorio con animales (ratas, palomas, etc) esta práctica se llama "análisis experimental de la conducta" (AEC) (Jaspiassu, 1981).

Esta práctica científica llamada AEC posee ciertas características distintivas. Según Skinner, la variable dependiente, que es la probabilidad de que una porción dada de conducta ocurra en un momento determinado se toma como dato natural en esta ciencia de la conducta. Esta probabilidad conductual se registra en términos de la frecuencia o tasa de respuesta (Catania, 1974).

En la práctica, las respuestas (o conductas) así definidas muestran una uniformidad conforme el organismo se mueve en un marco de referencia limitado por su propia anatomía y por el ambiente que lo rodea. Así pues, la tasa de respuesta que puede medirse con exactitud, constituye el eje de interés central de la "Ciencia de la Conducta".

Además debemos tener en cuenta, nos dice Skinner, que "la tarea del análisis experimental es describir todas las variables de las cuales es función la probabilidad de la respuesta" Se trata de las variables a las cuales Skinner denomina, por ejemplo: estímulos, ingestión de comida, administración de drogas, la edad del organismo, el peso del animal, las contingencias de reforzamiento (condicionamiento, extinción demora del reforzamiento, discriminación de estímulos, diferenciación de la respuesta, entre otras.

Según Skinner el AEC describe a los estímulos con el lenguaje de la Física. En este caso, al experimentador no le interesa sabe sí lo que para él es el estímulo lo es también para el animal. De ésta manera, conjeturar qué es lo que ve un organismo cuando se le muestra un estímulo y suponer que lo que se conjetura es lo que se le pregunta sería abandonar todo lo que la Física ofrece al especificar los acontecimiento ambientales.

Resumiendo, tenemos que los procesos conductuales que se estudian en el AEC consisten generalmente en cambios de probabilidad (o tasas de respuestas) que son función de las variables manipuladas (V.I); estas relaciones entre variables dependientes (V.D) y variables independientes (V.I) rara vez se han explorado bajo un diseño experimental, nos dice Skinner; la mayoría de veces los diseños se han hecho para probar hipótesis fisiológicas, mentalistas o conceptuales (Nudler, 1980).

\section{El aparato conceptual del AEC}

Basados en la metodología, arriba descrita, los psicólogos conductistas radicales se dedican a realizar experimentos en el laboratorio, utilizan su caja experimental y de algunas especies de animales. Las muchas horas de observación y de registro de la conducta de los sujetos en experimentación son descritas con un leguaje ingenioso, exacto y, sobre todo, "observable" y "medible"; es decir, construyen un lenguaje que describe "una a una" la diversidad de conductas en estudio. La aplicación del método inductivo es capital en esta tarea.

A partir de los hechos aislados se trata de realizar generalizaciones y por supuesto, acuñar ciertos principios o procedimientos que indican con "objetividad" ciertas regularidades naturales en el comportamiento de los sujetos en estudio.

$\mathrm{Al}$ respecto, Skinner se adhiere fundamentalmente a las descripciones operacionales estrictas de las experiencias, manteniendo cierta actitud de desconfianza frente a cualquier explicación técnica que vaya más allá de la conducta observable. Propone, a través de su artículo ¿Son necesarias las teorías del aprendizaje?, que las teorías son inútiles y perjudiciales ya que distraen al investigador del perfeccionamiento de sus experiencias conduciéndolos, la mayoría de las veces, por caminos improductivos o fomentando hipótesis de problemas que no puedan ser abordados experimentalmente.

Skinner plantea que los enunciados que conforman una teoría pertenecen a 3 tipos de territorios: uno donde los acontecimientos son observables, otros donde sólo tienen referencias observables y un tercero donde son inobservables. En esta distinción coincide con la división, hecha por Carnap, sobre los niveles de las teorías. Ahora bien ¿Cuál es el territorio o nivel de observación que toma Skinner para construir su 
sistema teórico?

La respuesta a esta pregunta está relacionada al uso que Skinnerdá al término "Control": se controla determinada respuesta, sí, mediante procedimientos determinados y específicos, se puede lograr que un organismo produzca, incremente, decremente o extinga dicha respuesta. El control puede conducir, además a conseguir determinadas formas, fuerza y duración de la respuesta. Según los conductistas radicales, el logro de este control depende de las medidas que se logre aislar y manipular de las variables de las cuales depende el hecho observado. El concepto "control" así planteado obliga a trabajar en ambientes capaces de ser manipulados, en el caso del sistema skinneriano, corresponde a las respuestas que se ejercen sobre el medio, a "hechos observables", y a las situaciones estimulatorias o "eventos observables", es decir, el nivel de referencia de Skinner para construir su sistema teórico es aquél donde las respuestas pueden ser controladas por medio de variaciones del estímulo (por ejemplo, programas de reforzamiento, programas de moldeamiento, castigo negativo, etc), que se ubican en ámbitos manipulables (el investigador lo puede manejar a voluntad).

En otras palabras, el nivel de observación que trabaja Skinner en su sistema psicológico, es aquel en el que, tanto el hecho que se observa como los eventos que se aducen para explicarlo, son directamente observables (Restrepo, 1974). Para Skinner sólo son relevantes los hechos que sí se desprenden de una proposición que las relacione unívocamente con cierto tipo de eventos, y que tal relación sólo es posible sí se trabaja en el mismo nivel de observación.

En virtud a las anteriores consideraciones es que se levantan los enunciados más relevantes, que poco a poco, van dando vida al lenguaje acuñado por el conductismo radical. Por ejemplo, los eventos o conceptos como: operantes, triple relación de contingencia; tasa de respuesta; reforzamiento negativo o positivo; moldeamiento; entre otros, son eventos que describen variables claramente definidas, de tal manera que, representan conceptos unívocos, esto es, no sujeto a diferentes interpretaciones. De lo contrario, según el sistema skinneriano, no se podrá asegurar la objetividad o inter objetividad del conocimiento. Cuando a un organismo se le coloca en una «caja experimental» (pongamos por caso a la rata albina) ésta emitirá varias respuestas de tal manera que cuando termina de dar una respuesta queda en posición de emitirla otra vez. La ratita (entre otras respuestas) presionará una palanca que se encuentra insertada al interior de la caja experimental.
Este hecho observado se define como operante o como «clase de respuesta por su efecto ambiental». Cuando la ratita no presiona la palanca o lo hace con poca frecuencia, se puede hacer que la presione con alta tasa o frecuencia, usando un procedimiento de moldeamiento, vale decir, con la «modificación gradual de cierta propiedad de las respuestas producidas por el reforzamiento de las aproximaciones sucesivas a cierto criterio que determina la clase operante que se pretende establecer». Llega un momento en que la ratita al percatarse que una luz se enciende, presiona la palanca $(\mathrm{R})$, se aproxima al comedero, coge un grano de alimento (ER) y lo come rápidamente.

Todo este proceso, que describe las relaciones que se dan en la caja experimental, entre la presión de palanca $(R)$ en presencia de una luz (ED) y, el de coger y comer alimento (ER), como consecuencia de la respuesta, se define bajo el concepto de triple de relación de contingencia. Una vez que la ratita se encuentra bajo la influencia de la triple relación de contingencia, la presión de palanca tenderá a incrementarse. Esta última descripción define claramente el concepto de la tasa de respuesta.

De otro lado, el concepto reforzamiento positivo, describe claramente el hecho de presionar la palanca y como consecuencia de ello recibir la comida (ER); el concepto de reforzamiento negativo, describe claramente el hecho de presionar la palanca $(\mathrm{R})$ y como consecuencia de ello, la terminación o posposición de un castigo o estímulo aversivo (EA). Por ejemplo, cuando se le transmite corriente eléctrica (EA) a la ratita y ésta tiene que presionar la palanca $(\mathrm{R})$ para que cese el flujo eléctrico (EA).

Lo mismo sucede con los demás eventos o conceptos del lenguaje conductista radical, a saber, los hechos conductuales son descritos por eventos o conceptos que se encuentran en un mismo nivel de observación, es decir, cada evento o concepto describe o define operacionalmente a los hechos. Así, en este sistema psicológico los conceptos: operante, reforzamiento positivo, etc. se definirán de la misma manera y no podrá dárseles diferentes interpretaciones, sino la que el concepto construido a partir de los hechos empíricos les proporcione.

\section{La predicción en el AEC}

La predicción de un hecho está relacionado directamente con el "modus tollens" y su aplicación a determinadas teorías, leyes o hipótesis, es decir, con aquella regla lógica donde el hecho que se produce es consecuencia lógica de la conjunción de una premisa 
general, con una premisa singular o particular. En efecto, las leyes e hipótesis en la ciencia se presentan en la forma general "si A, entonces B", esto es, formulaciones contra factuales, y por tanto, no afirman La existencia de fenómenos, sino condiciones, por lo que las leyes o hipótesis son equivalentes a la forma: "Si algo es A, entonces es B". Se deduce luego que para hacer una predicción será necesario afirmar la existencia de algo observable para que a partir de una ley o hipótesis derivemos otro hecho.

Según Restrepo (Tomado de: Ardila, 1974), un sistema teórico debe contar también con un cuerpo de definiciones que constituyan los términos observacionales y que sirvan justamente para "identificar" los fenómenos (eventos o hechos) que pertenecen a un tipo dado. Y, claro está, el sistema sirve así para afirmar la existencia de un evento: si "algo" cumple con las condiciones de una definición explicita en la misma teoría. Esta es, entre otras cosas, el principal papel de las definiciones operacionales en la ciencia.

En consecuencia, podemos formular otra característica de la predicción: una predicción será tanto más precisa cuanto más precisos sean sus definiciones y términos descriptivos. El problema de la predicción en el sistema skinneriano está íntimamente ligado al concepto de "control" (ya mencionado). Si se pueden aislar las variables de las cuales es función una respuesta, entonces tendremos más control sobre ellas y en consecuencia se podrá predecir con mayor seguridad la emisión de la respuesta deseada.

Estas consideraciones se pueden extender a todos los organismos, de los cuales captamos las causas de su comportamiento y (con las restricciones del caso) y por ende a los seres humanos también. En suma, para el AEC predicción es sinónimo de "control", cuanto menos sea el control que tengamos de las variables responsables de la emisión de respuestas menor será la predicción de dichas respuestas y viceversa.

\section{A modo de sintesis: Los PIC y el AEC}

Después de haber establecido las consideraciones fundamentales del paradigma epistemológico de los programas de investigación científica de Lakatos, y de haber dado una revisión al marco conceptual y metodológico del sistema psicológico skinneriano, queda por analizar el estatuto de ciencia de la propuesta conductista radical a la luz de las formulaciones epistemológicas propuestas por Lakatos.

\section{El "nucleo firme" en el análisis experimental de la conducta (AEC)}

Decidimos que los principios conductuales tales como: tasa de respuestas, triple relación de contingencia y reforzamiento son el núcleo firme de la propuesta skinneriana. Decisión en virtud de un acuerdo o consenso, entre conductistas radicales al sostener que estos conceptos son la piedra angular de su teoría. Cabe remarcar que, Lakatos no refiere criterios específicos a seguir para decidir sobre qué enunciados o qué principios de una teoría deben ser considerados como su "núcleo firme".

Así, el modelo ED-R-ER o triple relación de contingencia que se utiliza nos permite apreciar que las relaciones entre estos eventos están siempre presentes al hacer un análisis serio de las conductas. De no ser así nos hallaríamos fuera del contexto de la propuesta en mención. Del mismo modo sucede con el principio de reforzamiento en tanto que la pareja de variables respuesta estímulo reforzador (sea éste positivo o negativo) es clave para la descripción y comprensión de que la "respuesta es función de sus consecuencias". Dejar de lado éste principio nos conduciría, quizás, a los criterios de "reforzamiento" usados en el sistema de Pavlov o en el sistema de Hull (Ribes, 1985). La tasa de respuesta, según Skinner es el dato más relevante porque se encuentra íntimamente ligado al criterio de medición, que en ciencia, y sobre todo en psicología, es fundamental. Este criterio de "medición conductual" es típico del sistema skinneriano.

De otro lado, Lakatos exige que todo "programa de investigación científica" debe contar con un "núcleo firme" de conceptos o hipótesis básicos, es decir, el sustento de la teoría, pero que además, sean impermeables a los ataques del Modus Tollens lo que le daría su carácter de inamobible.

Ahora bien, ¿el sistema conceptual skinneriano cumple con esta primera exigencia que pide Lakatos? veamos, en su estado actual este sistema presenta una serie de limitaciones y de contradicciones que parecen sugerir nuevas formulaciones, fundamentadas en premisas que consideren el reajuste de algunos de sus supuestos que han guiado su desarrollo durante 50 años. En uno de sus trabajos de corte epistemológico, Emilio Ribes nos dice: "El papel central del concepto reforzamiento y de la tasa de respuesta como unidad de medida de la conducta, han restringido la investigación experimental en la búsqueda de efectos cuantitativos de incremento o decremento de formas particulares de 
conducta susceptibles de ser afectados de esta manera" (Ribes, 1985). Sin embargo, podemos apreciar que muchas de las conductas emitidas por el ser humano y por otros organismos son interacciones que no sólo se enmarcan dentro del contexto repetitivo o de alta o baja frecuencia de ocurrencias, sino que también se caracterizan por su alta precisión (más aún si se trata del ser humano). Este énfasis (en la tasa de respuesta) condujo a igualar, de forma incorrecta, la probabilidad de respuesta con su frecuencia.

En relación al concepto de la "triple relación de contingencia" se han producido una serie de anomalías relacionadas con la lógica que impone éste principio. Por ejemplo, a lo largo de los trabajos experimentales han aparecido una serie de hechos conductuales que escapan al concepto "triple relación de contingencia" para describirlos y comprenderlos. Esto produce una serie de paradojas, contradicciones y fenómenos (hasta ahora) huérfanos de concepto; me refiero específicamente a fenómenos observados como: "... los efectos reforzantes de los estímulos eléctricos; la posposición de alimento; los efectos cuantitativos de reforzadores no contingentes; los efectos mantenedores de tasas elevadas por estímulos breves; la imposibilidad de manipular los parámetros de demora; la dificultad de incorporar en un mismo marco interpretativo los fenómenos de variación local de la tasa (compensación, contraste, exploraciones, etc.), que trascienda la circularidad de un argumento matemático que define lo que incluye en su descripción; la reducción de la tasa ante los estímulos discriminativos y el aumento de la tasa ante los estímulos delta; etc." (Ribes, 1980).

En consecuencia, las anomalías, paradojas y excepciones continuarán apareciendo en la medida en que no se modifique el sistema conceptual productor de éstos datos, y que por razones diversas no le imprimen coherencia interna. Para RIBES, éste problema es de orden conceptual; para nosotros el problema va más allá del marco conceptual, depende, sobre todo, de la concepción o manera de concebir el fenómeno psíquico. "La teoría debe cambiar, sostiene RIBES, no solo para resolver irregularidades empíricas que se han generado bajo su orientación, sino también para abrir nuevos campos de investigación que puedan alterar la jerarquía valorativa de los datos actualmente contrastados" (Ribes, 1985).

Si el conjunto de conceptos o de hipótesis que hemos supuesto conforman el "núcleo firme" de la teoría skinneriana estas han sido rebatidas por el modus tollens $\mathrm{y}$, por tanto, se encuentra limitada $\mathrm{y}$ en medio de paradojas y contradicciones. Entonces podemos inferir, en una primera aproximación, que la teoría skinneriana: 1) no es una teoría consistente o de alto nivel; 2) no cuenta con un "núcleo firme" de conceptos o hipótesis y, 3) al ser cambiados o re conceptualizados tan sólo sus conceptos básicos, no lograría el estatus de teoría según las exigencias de LAKATOS, es decir, no lograría constituir, aún, un "programa de investigación científica". Se trataría, en todo caso, de un conjunto de conceptos, de principios empíricos que no permitirían, por ahora, vertebrar una real y sistematizada teoría científica psicológica.

\section{El cinturón protector en el AEC}

La propuesta en estudio se ubica más adecuadamente en este nivel. En la versión lakatosiana, a este nivel las hipótesis o conceptos cumplen un papel de auxiliares y se encuentran llenas de anomalías sobre cuya base se establecen las condiciones iniciales. Estos conceptos pueden ser refutados, modificados, expandidos. El sistema de Skinner sólo llega a cumplir con esta segunda exigencia que pide Lakatos para que se establezca un real «programa de investigación científica». En este nivel, actúan directamente los principios lógicos del modus tollens que al ser aplicados al interior del marco conceptual del conductismo radical se ha detectado problemas que se pueden ir solucionando con un ajuste adecuado de sus premisas básicas. Según LAKATOS, estas maniobras son ad hoc y deben de ir en consonancia con el aparato «heurístico» que se maneje (Lakatos, 1978).

El conductismo radical parece estar por esta vía. Muestra de ello se refleja en la obra de RIBES, donde trata de dar los primeros pasos en la sistematización de una «Teoría de la Conducta» basado en un análisis de campo y paramétrico, inicialmente trabajado por Kantor (1979).

Dejemos que Ribes (1985) nos aclare su propuesta: «la taxonomía que hemos elaborado se fundamenta, por ello, en el paradigma de KANTOR como opción válida ante las anomalías de la ciencia normal (alude a Khun) desarrollada por y gracias a Skinner. Como oposición conceptual no pretende agregar solamente nuevas categorías a las ya existentes, sino que significa un intento de sustitución de dichas categorías.

\section{Los factores "heuristicos" en el AEC}

Consideramos que el sistema de Skinner sí cuenta con los factores "heurísticos" o técnicas para solucionar sus problemas. Al respecto se puede 
mencionar la creación de una amplia gama de "diseños conductuales". Estos diseños conductuales experimentales le permiten al experimentador crear una diversidad de ambientes artificiales para el estudio del comportamiento de los organismos, o en términos de Ribes, para el estudio de la «interacción conductual» (Ribes, 1980).

También manejan una serie de técnicas de observación y registro. Sin embargo, nuestro estudio ha encontrado que al interior del AEC está actuando una especie de «heurística negativa» que se encarga de bombardear, a través de contrastaciones, los conceptos o hipótesis auxiliares. De otro lado, también parece estar actuando una «heurística positiva» que conduce a mecanismos de como modificar o complicar el «cinturón protector» que está siendo refutado. Este sería el caso, por ejemplo para el AEC, de la alternativa planteada por RIBES. Propuesta que se encontraría bajo el dominio de la «heurística positiva», de ahí pues, la selección racional que ha hecho de los problemas más acuciantes que atraviesa el AEC.

\section{Acerca de la predicción en el AEC}

Los conceptos de predicción en Skinner y en Lakatos son diferentes. Skinner plantea su definición dependiente totalmente de consideraciones factuales, restringiendo el término al "control experimental". Skinner cree que predecir una conducta es establecer cierta repetitividad de ella: si se logra aislar y controlar las variables de las cuales es función una conducta, entonces se puede predecir con alta certeza las condiciones y tipos de conducta que se presentarán en el futuro, pero, como repetición de conducta ya establecidas. La versión empírica de predicción no va más allá de los hechos observados o verificados; en tal sentido, de seguir este concepto con restricciones, de orden cualitativo, no podría esperarse que se realicen predicciones de gran envergadura.

En el sistema de Lakatos la predicción es ante todo la anticipación que hace la teoría sobre los hechos, es decir, la predicción va de la mano con el concepto de "problemática progresiva". En cambio, la "problemática estancada" va de la mano con el carácter no predictivo de las teorías, vale decir, cuando el desarrollo teórico queda rezagado respecto del desarrollo empírico. Según esta versión el sistema de Skinner se encontraría "estancado" y por tanto, es un sistema que carece de reales predicciones. Sin embargo, esta falta de predictividad, tal como la propone Lakatos, puede ser sólo temporal, dependerá en todo caso de los cambios que se produzcan en sus hipótesis auxiliares y de las maniobras ad hoc para proponer soluciones en la constitución de su "núcleo firme" que aún no posee.

\section{CONCLUSIONES}

Desde la perspectiva de Lakatos, el AEC no conforma aun un "programa de investigación científica" porque carece de principios o conceptos altamente estables y coherentes internamente que le permitan establecer el "núcleo firme" de su teoría.

Según los requerimientos que exige el paradigma lakatosiano para considerar a las teorías como científicas, el AEC sólo ha llegado a establecer conceptos a nivel del "cinturón protector", es decir, de rango auxiliar y no fundamental. Se ha establecido también que sí satisface el criterio de "heurística" como técnica para la solución de sus problemas.

El sistema skinneriano se encuentra en crisis cuyas causas no solamente se deben a problemas conceptuales, sino sobre todo, a una falta de revisión de sus fundamentos filosóficos (positivismo, pragmatismo, empirismo, etc.). De lo contrario, sus hallazgos experimentales seguirán en las anomalías ya analizadas porque no cuentan con la luz que guíen las explicaciones más cercanas a la realidad.

Al supeditar el concepto de predicción a los simples hechos factuales, el AEC tendrá muchos problemas para que su teoría sea admitida como científica. Desde la perspectiva lakatosiana las exigencias para decidir sobre le carácter científico de un sistema de enunciados se funda, antes que nada, en la "predicción de hechos nuevos", hechos que ni siquiera previamente se habían soñado y según hemos analizado el criterio de predictividad que tiene Skinner no va por ese camino.

Este trabajo ha querido contrastar, bajo la luz del modelo lakatosiano, el estatus de ciencia del sistema skinnriano. Este contraste y los resultados obtenidos, nos conducen a afirmar que sí es posible realizar, hasta cierto nivel, un análisis epistemológico de los paradigmas psicológicos contemporáneos.

El presente artículo fue escrito el año 1997. Fu revisado el 2010 para el curso: Epistemología de la Psicología del Programa de Doctorado en Psicología de la Facultad de Psicología Leopoldo Chiappo Galli. Universidad Peruana Cayetano Heredia. Lima, Perú. 


\section{REFERENCIAS BIBLIOGRÁFICAS}

1. Catania, Ch. (1974). Investigación contemporánea en conducta operante. México DF: Trillas.

2. Fernandez, G \& Natalicio, L.F. (1975). La ciencia de la conducta. México DF: Trillas.

3. Kantor, J. R. (1979). Psicología Interconductual. México DF: Trillas.

4. Jaspiassu, H. (1981). Introducción a la Epistemología de la Psicología. Bogotá: Universidad Santo Tomás, Centro de enseñanza desescolarizada.

5. Kedrov, B.M. (1977). Filosofía y ciencias naturales. Ciencias Sociales, 3.

6. Kuhn, T. (1977). La estructura de las revoluciones cientificas. México DF: FCE.

7. Lakatos, I. (1974). Historia de la ciencia y sus reconstrucciones racionales. Madrid: Tecnos.

8. Lakatos, I. (1978). La metodología de los programas de investigación científica. Madrid: Alianza Universidad.

9. Mikulinski, S. (1977). Estado actual y problemas teóricos de la historia de las ciencias naturales. Revista Problemas del mundo contemporáneo, 49.

10. Nudler, O. (1980). Epistemología de la Psicología. México DF: Trillas.
11. Paniagua, F. (1986). Synthetyc behaviorism: remarks on funtion and structure. Psychology Record, 36(2), 179-184.

12. Piaget, J. (1978). Historia de la Psicología Experimental. México DF: Trillas.

13. Piaget, J. (1978). Naturaleza y métodos de la Epistemología. Buenos Aires: Paidós.

14. Popper, K. (1978). El psicoanálisis entre el mito y la ciencia. En: D. Antiseri. Análisis epistemológico del marxismo y el psicoanálisis. Salamanca: Ediciones Sígueme.

15. Popper, K. (1980). La lógica de la investigación cientifica. Madrid: Tecnos.

16. Pray, L. (2008) Discovery of DNA structure and function: Watson and Crick. Nature Education, 1(1),100.

17. Restrepo, H. (1974). El condicionamiento operante a la luz de la filosofía de la ciencia. En: R. Ardila. El análisis experimental del comportamiento: la contribución Latinoamericana. México DF: Trillas.

18. Ribes, E. (1980). Reflexiones criticas sobre conductismo. Barcelona: Fontanella.

19. Ribes, I. (1985).Teoría de la conducta. México DF: Trillas.

20. Robinson, D. (1985). Historia crítica de la Psicología. Barcelona: Salvat. 